\title{
Medición de presiones relativas en aorta torácica y arteria pulmonar de voluntarios sanos y pacientes con Tetralo- gía de Fallot reparada utilizando la secuencia 4D Flow de resonancia magnética cardíaca
}

Dres. Jesús Urbina $R^{(1,2)}$, Julio Sotelo $P^{(2,3)}$, Cristián Tejos $N^{(2,3)}$, Daniel Hurtado $S^{(2)}$, Marcelo Andía K(2,4), Pablo Irarrázaval $M^{(2,3)}$, Sergio Uribe $A^{(2,4)}$.

1. Facultad de Medicina, Pontificia Universidad Católica de Chile. Santiago - Chile.

2. Centro de Imágenes Biomédicas, Pontificia Universidad Católica de Chile. Santiago - Chile.

3. Departamento de Ingeniería Eléctrica, Pontificia Universidad Católica de Chile. Santiago - Chile.

4. Departamento de Radiología, Facultad de Medicina Pontificia Universidad Católica de Chile. Santiago - Chile.

Relative pressure measurement in thoracic aorta and pulmonary artery in healthy volunteers and patients with Tetralogy of Fallot repaired by 4D Flow cardiovascular MRI

Abstract: Objective. To validate the utility of $4 D$ Blood Flow and Navier-Stokes equations to create relative
pressure (RP) maps in the aorta and pulmonary artery (PA) in healthy volunteers and patients with repaired
tetralogy of Fallot (TOF). Methods. A $4 D$ flow sequence of whole heart and its major vessels was acquired
in 10 healthy volunteers and 6 patients with repaired TOF. The root of the ascending aorta was used as
the reference point to calculate RP along five different points of this area. In addition, relative pressure of
both right and left PA was measured as correlated to absolute pressure. Results. Patients with repaired
TOF showed greater pulmonary artery $(P A)$ relative pressure differences between maximum and minimum
values when compared to volunteers $(P<0.05)$. Additionally, aortic relative pressures had an excellent co-
rrelation with published data, whether using $4 D$ flow or by catheterization. Conclusions. $4 D$ Flow MRI may
represent a new non-invasive and non operator-dependent diagnostic tool in $C V$ disease management.
Key words: $4 D$ Flow, Congenital heart defects, Magnetic resonance imaging, Relative pressure difference.

Resumen: Objetivo. Utilizar 4D Flow y las ecuaciones de Navier-Stokes para obtener mapas de presiones relativas $(P R)$ en la Aorta y Arteria Pulmonar $(A P)$ de voluntarios y pacientes con Tetralogía de Fallot reparada (TOFr). Métodos. En 10 voluntarios y 6 pacientes con TOFr se adquirió la secuencia 4D flow del corazón y sus principales vasos. La raíz de la Aorta Ascendente se utilizó como referencia para calcular las $P R$ a esta zona en cinco puntos distintos. Además, se midió la PR de la AP derecha e izquierda respecto a la $A P$. Resultados. Los pacientes con TOFr tuvieron diferencias de $P R$ entre los valores máximos y mínimos más grandes que los voluntarios en la $A P(p<0,05)$. Adicionalmente, las $P R$ de la aorta tuvieron una excelente correlación con datos publicados utilizando 4D flow y mediante cateterización. Conclusiones. 4D Flow podría constituir una nueva herramienta diagnóstica, no invasiva, ni operador dependiente, en el manejo de patologías CV.

Palabras clave: 4D flow, Cardiopatía congénita, Diferencia de presiones relativas, Resonancia magnética.

Urbina J, et al. Medición de presiones relativas en aorta torácica y arteria pulmonar de voluntarios sanos y pacientes con Tetralogía de Fallot reparada utilizando la secuencia 4D Flow de resonancia magnética cardíaca. Rev Chil Radiol 2012; 18(4): 157-162.

Correspondencia: Dr. Sergio Uribe A. / suribe@med.puc.cl

Primer premio en trabajos orales Congreso Chileno de Radiología 2012.

\section{Introducción}

Las gradientes de presiones son un importante marcador clínico para determinar la severidad de distintas patologías cardiovasculares (PC), como cardiopatías congénitas ${ }^{(1)}$, estenosis valvulares ${ }^{(2,3)}$ y coartación aórtica ${ }^{(4)}$. En la práctica clínica habitual la presión es medida mediante un esfigmomanómetro, el cual entrega una generalizada estimación de la 
presión sistólica y diastólica absoluta en un punto espacial del sistema cardiovascular periférico. Sin embargo, el estándar de oro para medir presiones en los grandes vasos es el cateterismo cardíaco, técnica invasiva que irradia al paciente, no exenta de riesgos y no fácilmente reproducible.

Se ha estudiado extensamente la obtención de gradientes de presiones y presiones relativas (PR) a través de la medición de velocidades en el tiempo en los vasos sanguíneos. La ecocardiografía es utilizada para estimar presiones en forma indirecta midiendo velocidades máximas y utilizando la ecuación de Bernoulli. Sin embargo, posee limitaciones tales como malas ventanas acústicas, es operador dependiente, es altamente sensible a las velocidades máximas medidas debido a la exponenciación de esta variable y sus valores son sobre-estimados respecto a los medidos por cateterismo ${ }^{(5)}$. Además, esta técnica no toma en cuenta los cambios de presión producidos por la aceleración de la sangre, su fricción con la pared y la distensibilidad de los vasos ${ }^{(6)}$, por lo que no puede ser utilizada para medir pequeños cambios de presión o para flujos complejos ${ }^{(11)}$, lo que es importante en la evaluación de niños con cardiopatías congénitas.

Se ha demostrado en resonancia magnética que es posible obtener también estimaciones de presiones a partir de datos de velocidad a lo largo del tiempo. Mas aún, los datos obtenidos a partir de la secuencia 4D Flow (datos de velocidad obtenidos en tres dimensiones espaciales y una dimensión temporal) pueden ser utilizados para obtener mapas relativos de presión ${ }^{(7-14)}$. Esto es posible de realizar simulando tridimensionalmente las ecuaciones de Navier-Stokes que relacionan las derivadas de la velocidad con los gradientes de presión ${ }^{(10,14)}$.

El objetivo de este trabajo es utilizar la secuencia 4D Flow y la modelación de las ecuaciones de NavierStokes para obtener mapas de presiones relativas en la aorta (Ao) torácica y arteria pulmonar (AP) de voluntarios sanos y pacientes con Tetralogía de Fallot reparada (TOFr) en forma no invasiva, con el fin de demostrar la utilidad clínica de 4D Flow como nueva herramienta diagnóstica en la medición de presiones. De esta forma uno podría mejorar la comprensión y manejo clínico de las patologías cardiovasculares.

\section{Material y métodos}

En 10 voluntarios sanos, edad promedio 32,1 $\pm 6,6$ años (rango de edad: $24-45,7$ hombres) y 6 pacientes con TOFr, edad promedio 16,5 $\pm 5,2$ años (rango de edad: 11-25, 2 hombres) se realizó una resonancia magnética cardíaca (RMC) estándar con un resonador Philips 1.5T (Philips, Healthcare, Best, The Netherlands). El protocolo incluyó una secuencia de contraste de phase 2D en la aorta, arteria pulmonar, arteria pulmonar derecha (APD) y arteria pulmonar izquierda (API). Además se incluyó una secuencia
4D Flow del corazón y de los principales vasos del mediastino. Para la secuencia 4D Flow se utilizaron los siguientes parámetros de adquisición: 50 cortes, ángulo de excitación de $6^{\circ}$, resolución temporal de 38 $\mathrm{ms}$, resolución espacial de $2.5 \mathrm{~mm}^{3}$ y VENC $=200$ $350 \mathrm{~cm} / \mathrm{s}$. Con esta técnica se obtuvo una imagen anatómica y tres imágenes de velocidad, una por cada uno de sus vectores $(X, Y, Z)$.

\section{Obtención de mapas de presión}

Los datos de 4D Flow fueron visualizados y procesados a través del software comercial GTFlow 2.0.4 (Gyrotools LCC, Zurich, Suiza). Se generaron máscaras para obtener las regiones de interés con la aorta torácica y la arteria pulmonar por separado. Una vez obtenidos los vasos de interés aislados, se generó un mapa de presiones relativas utilizando la ecuación de Navier-Stokes asumiendo un fluido viscoso, laminar e incompresible:

$$
\nabla^{2} \mathbf{p}=\nabla\left(-\rho \frac{\partial V}{\partial t}-\rho V \cdot \nabla V+\mu \nabla^{2} V\right)
$$

V: Velocidad, p: presión, $\rho$ : densidad del fluido, $\mu$ : viscosidad del fluido

Para el cálculo de las presiones relativas (PR), se utilizó la raíz de la aorta ascendente (AAs) como referencia para calcular PR a este punto en: (I) AAs a la altura del tronco pulmonar, (II) AAs antes del tronco braquiocefálico, (III) después de la arteria subclavia izquierda, (IV) en la aorta descendente (ADs) a la altura del punto de referencia y (V) en la ADs $5 \mathrm{~cm}$ bajo el punto de referencia. Además, se midió la presión de la AP derecha e izquierda (APD y API) inmediatamente antes de sus ramificaciones, respecto al tronco principal de la AP.

\section{Análisis de los datos}

Las diferencias máximas de presión de cada individuo obtenidas en la Ao y AP, fueron comparadas entre voluntarios y pacientes con TOFr en cada punto de medición a través de una prueba t de Student. Además, se analizaron los promedios de las presiones relativas en cada fase del ciclo cardíaco en la Ao y AP de voluntarios y pacientes con TOFr. Por último, los datos de la aorta torácica se compararon con valores publicados en la literatura obtenidos mediante cateterización. De acuerdo a una revisión del estado del arte hasta la fecha no existen valores de presión medidos mediante cateterización cardíaca en pacientes con TOFr.

\section{Resultados}

Un mapa de presiones relativas obtenido en un voluntario sano a lo largo del ciclo cardíaco (CC) se puede observar en las figuras 1 y 2 para la aorta y arteria 
pulmonar respectivamente. Las tonalidades café, roja y naranja representan los máximos valores de presiones, los cuales se alcanzan tempranamente, transcurrido el
$25 \%$ del ciclo cardíaco aproximadamente. Además, es posible observar algunas variaciones de las presiones relativas en un mismo segmento transversal de arteria.
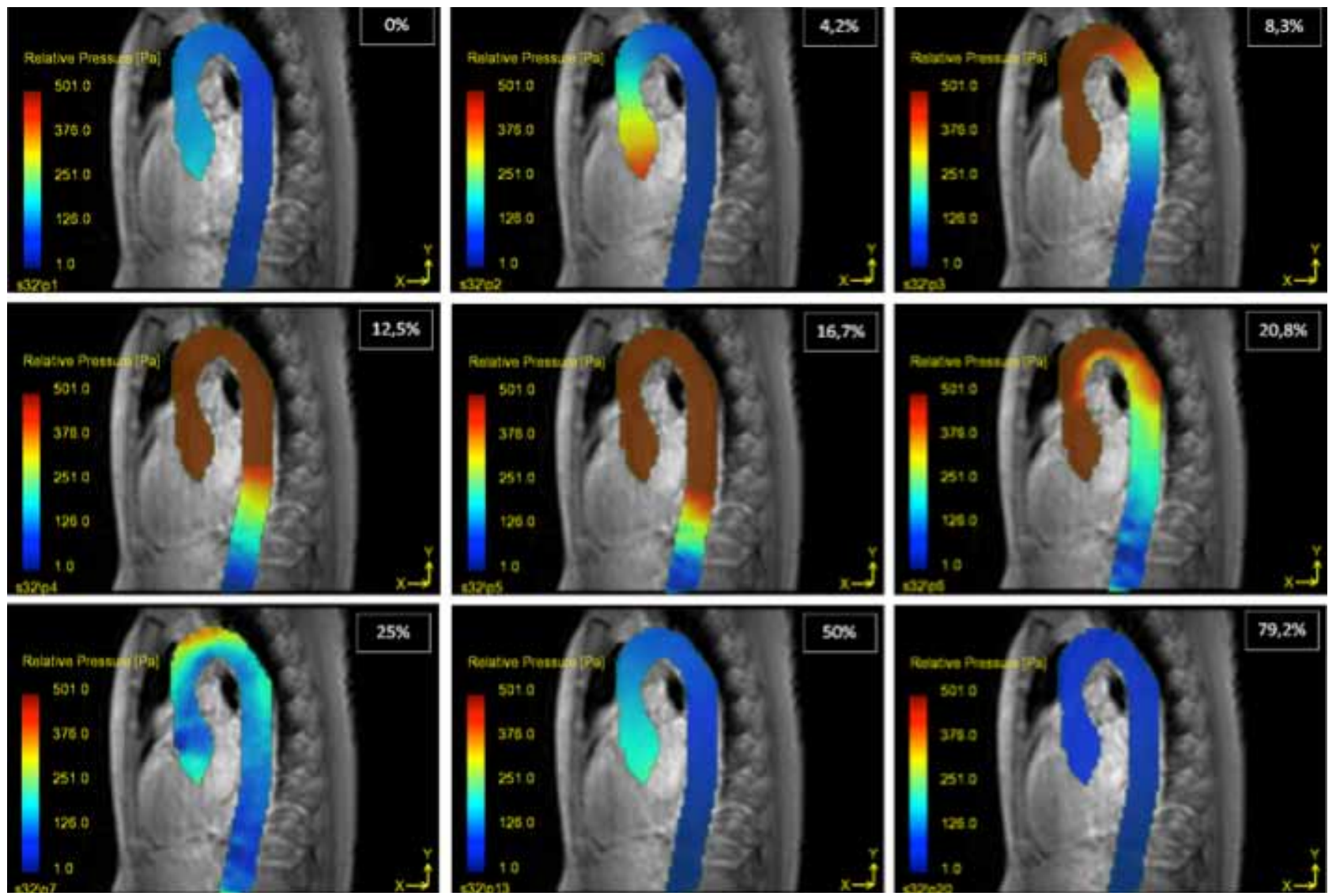

Figura 1. Gradientes de presiones en la aorta de un voluntario en distintas fases del ciclo cardíaco, a través de un corte sagital. Las presiones están indicadas en Pascales ( $P a)$. Las distintas fases cardíacas se expresan en porcentaje del ciclo cardíaco en la esquina superior derecha de cada imagen.
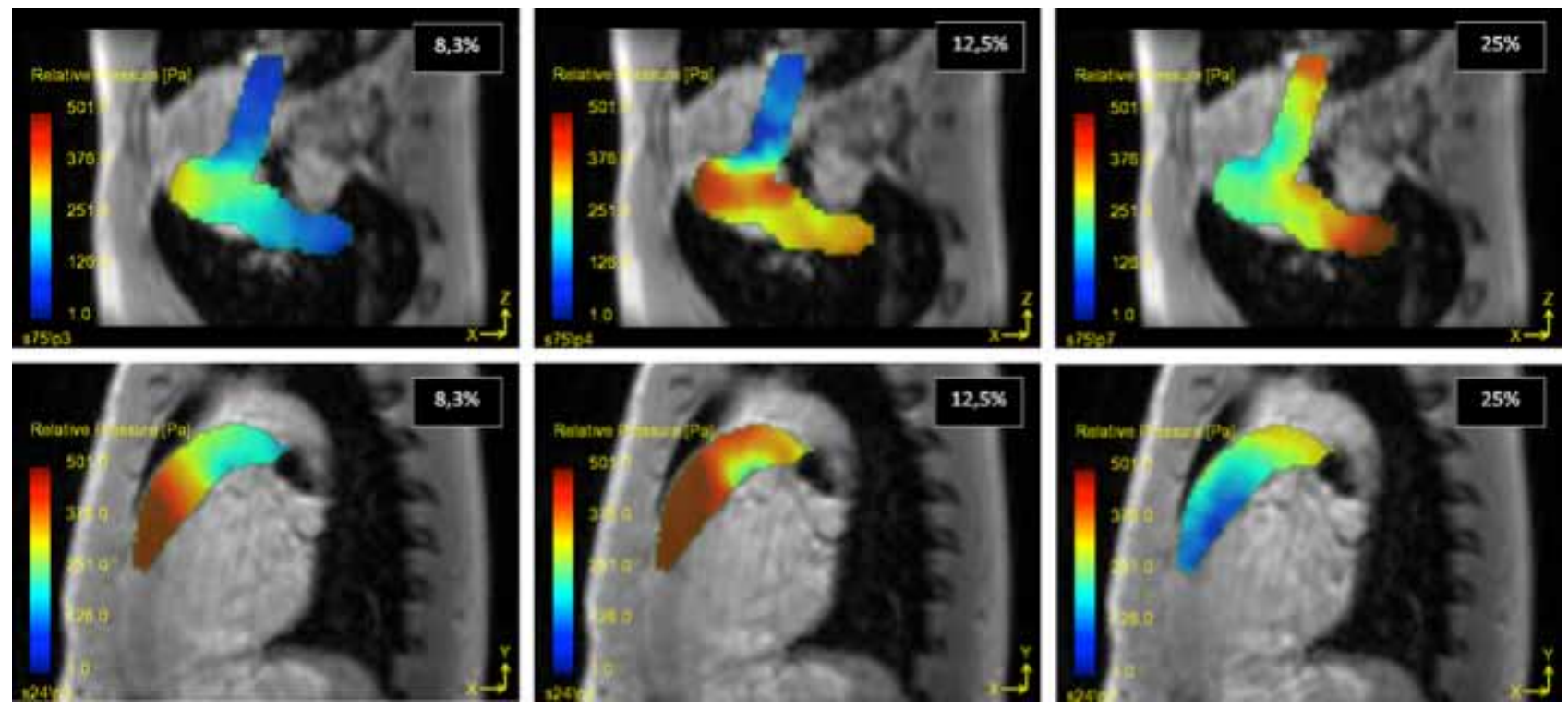

Figura 2. Gradientes de presiones en la arteria pulmonar de un voluntario en dos cortes distintos: las tres primeras figuras en corte transversal a nivel de la APD y API y las tres últimas figuras en un corte sagital a nivel del tronco pulmonar. Se muestran tres fases cardíacas distintas y se expresan en porcentaje del ciclo cardíaco en la esquina superior derecha de cada imagen. Las presiones están indicadas en Pascales $(\mathrm{Pa})$. 
La figura 3 muestra las curvas de presiones relativas para distintos puntos a lo largo de la aorta, obtenidas a partir del promedio en cada fase del CC, de 10 voluntarios y 6 pacientes con TOFr. La forma de la curva de voluntarios y pacientes es similar, sin embargo, los pacientes alcanzan en promedio más tardíamente los peaks sistólicos $(31,7 \pm 8,1 \%$ del CC) respecto a los voluntarios $(24,2 \pm 1,9 \%$ del CC). Además, los voluntarios obtuvieron peaks de PR más altos que los pacientes durante el sístole y el diástole, por tanto, la diferencia máxima de presiones en la aorta, entre sístole y diástole, fue más grande en promedio en los voluntarios que en los pacientes con TOFr.

La figura 4 muestra las presiones relativas entre el tronco de la arteria pulmonar y sus ramas. La forma de la curva de la arteria pulmonar es notoriamente distinta entre voluntarios y pacientes, no existiendo en estos últimos la característica onda negativa de la fase temprana del diástole. Los valores sistólicos fueron mayores en los pacientes durante todo el ciclo cardíaco, sin embargo, durante el diástole los valores fueron similares, así, la diferencia máxima de presiones en la AP, entre sístole y diástole, fue más grande en los pacientes que en los voluntarios, inverso a lo ocurrido en la aorta.

En las tablas I y II se describen los promedios de las diferencias máximas de presión obtenidos en cada individuo en los 5 puntos de la aorta y en la APD y API respectivamente, de pacientes sanos y con TOFr. Al comparar estos datos, los voluntarios obtuvieron valores más altos que los pacientes durante el ciclo cardíaco, sin embargo, estas cifras no son estadísticamente significativas ( $p>0,05$ ).

Al comparar el promedio de las diferencias máximas de presión en la AP de voluntarios con pacientes con TOFr, estos últimos obtuvieron peaks más altos (APD: $9,3 \mathrm{mmHg}$ y API: $9,5 \mathrm{mmHg}$ ) que los voluntarios (APD: $6,4 \mathrm{mmHg}$ y API: $5,7 \mathrm{mmHg}$ ) durante el CC y estas diferencias son estadísticamente significativas, $p<0,05)$.

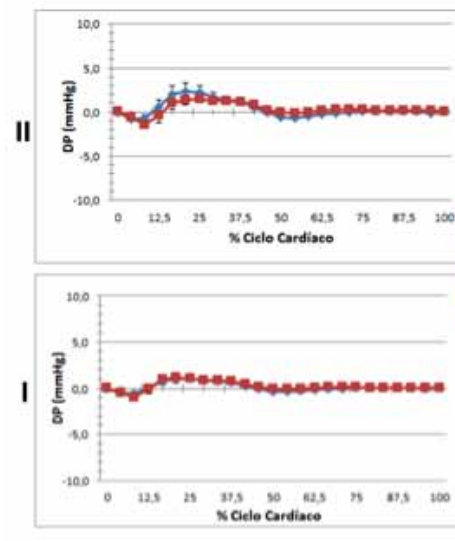

Voluntarios Sanos TOFr
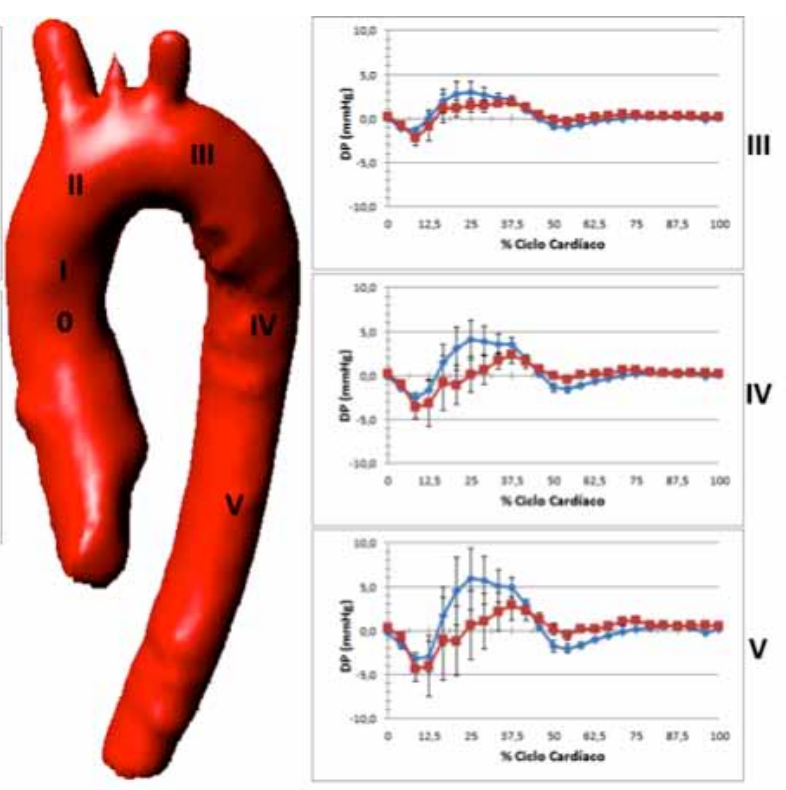

Figura 3. Aorta torácica de un voluntario que indica el punto de referencia (0) y las zonas donde se realizaron las mediciones. En cada punto se muestra la curva de presiones relativas obtenida en promedio con los 10 voluntarios y 6 pacientes con TOFr a lo largo de cada fase del ciclo cardíaco, el cual está graficado respecto al porcentaje de su duración total. En color azul están representadas las curvas de los voluntarios y en color rojo las curvas de los pacientes. DP: Diferencia de presión.

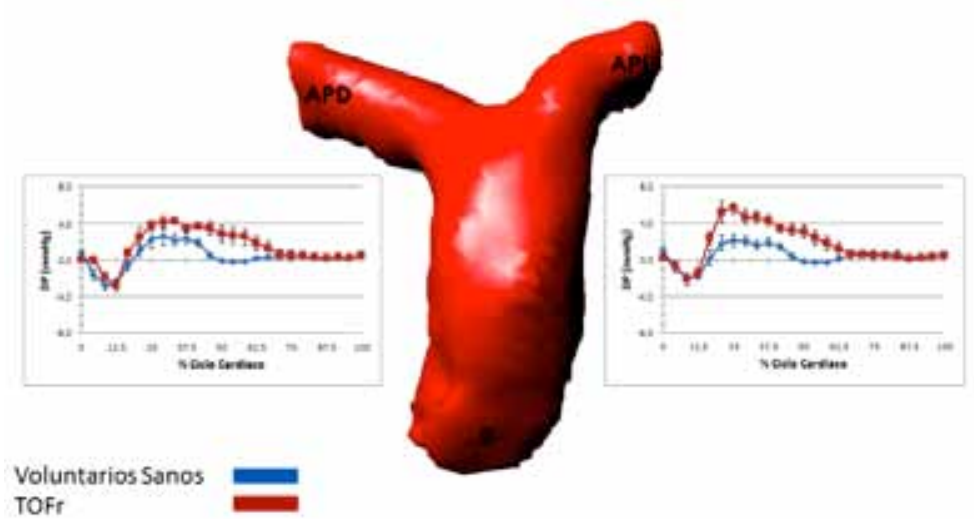

TOFr
Figura 4. Presiones relativas en la arteria pulmonar de un voluntario. La figura indica el punto de referencia (0) y las zonas donde se realizaron las mediciones ( $A P D$ y $A P I)$. El CC está graficado respecto al porcentaje de su duración total. En color azul están representadas las curvas de los voluntarios y en color rojo las curvas de los pacientes. DP: Diferencia de presión. 
Tabla I. Cuadro que muestra el promedio de las diferencias máximas de presión de cada individuo de la aorta de voluntarios y pacientes con TOFr. Los valores están expresados con una desviación estándar y con el rango en las cinco posiciones donde se realizaron mediciones respecto al punto de referencia. NS: Sin significancia estadística. Vol: Voluntarios.

\begin{tabular}{|c|c|c|c|}
\hline $\begin{array}{l}\text { Posición } \\
\text { en la Ao }\end{array}$ & & t-test & $\begin{array}{l}\text { Diferencia Máxima } \\
\text { de Presiones }\end{array}$ \\
\hline I & $\begin{array}{l}\text { Vol } \\
\text { TOFr }\end{array}$ & NS & $\begin{array}{l}2,5 \pm 0,9 \\
(1,7-4,1) \\
2,6 \pm 0,5 \\
(2,1-3,4)\end{array}$ \\
\hline II & $\begin{array}{l}\text { Vol } \\
\text { TOFr }\end{array}$ & NS & $\begin{array}{c}4,7 \pm 2,0 \\
(2,1-8,1) \\
4,0 \pm 1,6 \\
(2,9-6,8)\end{array}$ \\
\hline III & $\begin{array}{l}\text { Vol } \\
\text { TOFr }\end{array}$ & NS & $\begin{array}{c}6,7 \pm 2,7 \\
(3,6-11,7) \\
5,7 \pm 4,0 \\
(2,1-12,1)\end{array}$ \\
\hline IV & $\begin{array}{l}\text { Vol } \\
\text { TOFr }\end{array}$ & NS & $\begin{array}{c}10,3 \pm 4,1 \\
(5,2-19,8) \\
9,7 \pm 7,0 \\
(3,0-20,8)\end{array}$ \\
\hline V & $\begin{array}{l}\text { Vol } \\
\text { TOFr }\end{array}$ & NS & $\begin{array}{c}14,8 \pm 6,6 \\
(7,5-31,1) \\
15,6 \pm 8,2 \\
(5,2-26,7)\end{array}$ \\
\hline
\end{tabular}

\section{Discusión}

En este trabajo hemos utilizado datos de la secuencia 4D flow para obtener presiones relativas de presiones en la Aorta y Arteria pulmonar de voluntarios y pacientes con TOFr. La evidencia de este y otros estudios previos demuestran que esta técnica podría constituir una herramienta no invasiva ni operador dependiente para medir presiones en el estudio de patologías cardiovasculares.

En nuestro estudio encontramos que en la aorta de voluntarios y pacientes, la forma de la curva construida con el promedio de las presiones relativas en cada fase del CC fue similar, lo que se correlaciona en cierta medida con la similitud en su anatomía normal, sin embargo, los pacientes tienen peaks sistólicos más tardíos. Además, los voluntarios tienen peaks sistólicos y diastólicos más grandes. Esta tendencia
Tabla II. Cuadro que muestra el promedio de las diferencias máximas de presión de la arteria pulmonar de voluntarios y pacientes con TOFr. Los valores están expresados con una desviación estándar y con el rango en las dos posiciones de la AP donde se realizaron las mediciones respecto al punto de referencia. Vol: Voluntarios.

\begin{tabular}{|llcc|}
$\begin{array}{l}\text { Posición } \\
\text { en la AP }\end{array}$ & t-test & $\begin{array}{c}\text { Diferencia Máxima } \\
\text { de Presiones }\end{array}$ \\
\hline APD & Vol & & $\begin{array}{c}6,4 \pm 1,5 \\
(4,3-9,0) \\
\end{array}$ \\
& TOFr & $p<0,05$ & $\begin{array}{c}9,3 \pm 2,4 \\
(6,2-12,4)\end{array}$ \\
& & & $5,7 \pm 2,0$ \\
API & Vol & & $(3,0-9,2)$ \\
& & $p<0,05$ & $9,5 \pm 3,4$ \\
& TOFr & & $(6,0-14,9)$ \\
& & & \\
\end{tabular}

al ser analizada mediante una prueba t de Student, no tuvo significancia estadística.

En la AP, la forma de la curva de voluntarios y pacientes es distinta, siendo en esta última con valores sistólicos más altos $(p<0,05)$ y sin la característica onda negativa de la fase temprana del diástole. Estas diferencias podrían ser explicadas por las variaciones anatómicas propias de la TOF y las variaciones postquirúrgicas. Estas observaciones podrían constituir un nuevo marcador de seguimiento o para determinar la severidad de esta patología cardiovascular.

Si bien existe diferencia de edad entre los voluntarios sanos y los pacientes con TOFr, en este trabajo se compararon variaciones de presión entre dos puntos de la Ao y AP (presiones relativas) y no presiones absolutas. La presión absoluta puede ser distinta, pero el cambio de presión entre dos puntos, no debería ser significativamente distinta en ambos grupos de estudio. Por otro lado hubiésemos querido contar con adquisiciones de 4D Flow de los pacientes antes de que la Tetralogía de Fallot fuera reparada, para poder establecer comparaciones hemodinámicas antes y después de la operación, sin embargo, esto es difícil por la condición misma de estos pacientes.

Otra limitación del estudio es que no se poseen mediciones de presiones por cateterismo. Sin embargo, se intentó comparar los resultados obtenidos con datos publicados en la literatura. Mills ${ }^{(15)}$, en los años 70 realizó una serie de mediciones en la aorta y sus ramas utilizando cateterización. La única medición útil comparable con nuestro estudio, midió las presiones absolutas en la aorta ascendente y la aorta descendente, comparables a los puntos I y $\vee$ y que al 
calcular la diferencia de presiones se obtiene un valor de $8 \mathrm{mmHg}$, comparable a los $9,1 \mathrm{mmHg}$ obtenido en este trabajo mediante 4D Flow. También en los últimos años otros autores han publicado un reducido número de valores de presiones relativas obtenidas mediante 4D Flow. Tyszka ${ }^{(12)}$ publicó valores máximos y mínimos de 11/-5 medidos entre los puntos I y $\mathrm{V}$ aproximadamente. Bock ${ }^{(16)}$, en posiciones similares publicó valores de $7 /-3$, los cuales son cercanos a los valores obtenidos en nuestros voluntarios $(5,9 /-3,2)$. Entre los puntos II y III, Ebbers ${ }^{(14)}$ publicó valores máximos y mínimos de 3/-2, Nagao ${ }^{(17)}$ de 1/-0,6 y Bock et al publicó valores de 2/-1. Estos valores son similares a los nuestros $(0,6 /-0,6)$. Es importante considerar que los estudios realizados por Tyszka, Ebbers y Nagao sólo consideraron a un voluntario y los estudios de Bock a 12 voluntarios, por lo que los valores obtenidos por este último grupo son más comparables a nuestro trabajo.

\section{Perspectivas Futuras}

Con el fin de tener una mejor comprensión hemodinámica en pacientes con cardiopatías congénitas, es necesario analizar cuantitativamente la relación existente entre los flujos y los gradientes de presiones obtenidos mediante 4DFlow en experimentos controlados. En la actualidad estamos validando un fantoma de aorta torácica con la finalidad de facilitar las mediciones de flujos y presiones, y correlacionar estas últimas con valores obtenidos mediante cateterización. Por otro lado se podrá simular patologías cardiovasculares como estenosis aórticas y aneurismas de aorta torácica.

\section{Agradecimientos}

Los autores de este artículo agradecen al proyecto Fondecyt 11100427, ACT 079, y a la Vicerrectoría de Investigación de la Pontificia Universidad Católica de Chile por financiar este trabajo.

\section{Bibliografía}

1. Robinson S. Diagnosis of congenital heart disease: clues from the history and physical examination. Cardiovasc Clin 1970; 2 (1): 77-95.

2. Hatle L, Brubakk A, Tromsdal A and Angelsen B. Noninvasive assessment of pressure drop in mitral stenosis by Doppler ultrasound. Br Heart J 1978; 40: 131-140.

3. Rao P and Carey P. Doppler ultrasound in the prediction of pressure gradients across aortic coarctation. Am Heart J 1989; 118: 299-307.

4. Houston A, Simpson I, Pollock J, Jamieson M, Doig W,
Coleman E. Doppler ultrasound in the assessment of severity of coarctation of the aorta and interruption of the aortic arch. Br Heart J 1987; 57(1): 38-43.

5. Weber G, Strauss A, Rieger H, Scheffler A, Eisenhoffer J. Validation of Doppler measurement of pressure gradients across peripheral model arterial stenosis. J Vasc Surg 1992; 16: 10-16.

6. Stewart S, Nast E, Arabia F, Talbot T, Proschan M, Clark R. Errors in pressure gradient measurement by continuous wave Doppler ultrasound-type, size and age effects in bioprosthetic aortic valves. J Am Coll Cardiol 1991; 18: 769-779.

7. Sondergaard L, Stahlberg F, Thomsen C, Stensgaard $\mathrm{A}$, Lindvig $\mathrm{K}$ and Henriksen. Accuracy and precision of MR velocity mapping in measurement of stenotic cross-sectional area, flow rate, and pressure gradient. J Magn Reson Imaging 1993; 3: 433-437.

8. Urchuk S, Plewes D. MR measurement of pulsatile pressure-gradients. J Magn Reson Imaging 1994; 4: 829-836.

9. Urchuk $S$ and Plewes D. MR measurement of timedependent blood pressure variations. J Magn Reson Imaging 1995; 5: 621-627.

10. Yang G, Kilner P, Wood N, Underwood S and Firmin D. Computation of flow pressure fields from magnetic resonance velocity mapping. Magn Reson Med 1996; 36: 520-526.

11. Tyszka J, Laidlaw D and Silverman J. Relative pressure mapping using high-speed three-dimensional phase contrast cine MR imaging. Radiology 1997; 205: 304.

12. Tyszka J, Laidlaw D, Asa J and Silverman J. ThreeDimensional, Time-Resolved (4D) Relative Pressure Mapping Using Magnetic Resonance Imaging. J Magn Reson Imaging 2000; 12: 321-329.

13. Tasu J-P, Mousseaux E, Delouche A, Oddou C, Jolivet $O$ and Bittoun J. Estimation of Pressure Gradients in Pulsatile Flow From Magnetic Resonance Acceleration Measurements. Magn Reson Med 2000; 44: 66-72.

14. Ebbers $T$, Wigström L, Bolger $A$, Engvall $J$ and Karlsson M. Estimation of Relative Cardiovascular Pressures Using Time-Resolved Three-Dimensional Phase Contrast MRI. Magn Reson Med 2001; 45: 872-879.

15. Mills C, Gabe I, Gault J, Mason D, Ross J, Braunwald $E$, et al. Pressure-flow relationships and vascular impedance in man. Cardiovasc Res 1970; 4: 405-417.

16. Bock J, Frydrychowicz A, Lorenz R, Hirtler D, Barker A, Johnson K, et al. In Vivo Noninvasive 4D Pressure Difference Mapping in the Human Aorta: Phantom Comparison and Application in Healthy Volunteers and Patients. Magn Reson Med 2011; 66: 1079-1088.

17. Nagao T, Yoshida K, Okada K, Miyazaki S, Ueguchi T, Murase K. Development of a Noninvasive Method to Measure Intravascular and Intracardiac Pressure Differences Using Magnetic Resonance Imaging. Magn Reson Med Sci 2008; 7(3): 113-122. 